\title{
Perimenopausal women show modulation of excitatory and inhibitory neuromuscular mechanisms
}

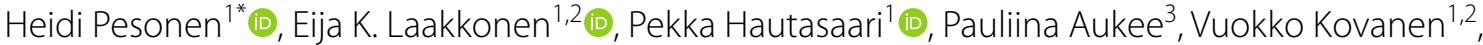 \\ Sarianna Sipilä ${ }^{1,2}$, Taija Finni ${ }^{1}$ and Ina M. Tarkka ${ }^{1}$ (D)
}

\begin{abstract}
Background: Menopausal transition exposes women to an early decline in muscle force and motor function. Changes in muscle quality and function, especially in lower limbs, are crucial, as they expose individuals to increased risk of falls. To elucidate some of the related neuromuscular mechanisms, we investigated cortical inhibition and peripheral muscle twitch force potentiation in women during the early and late stages of perimenopause.

Methods: Participants were 63 women aged $48-55$ years categorized as early $(E P, n=25)$ or late $(L P, n=38)$ perimenopausal according to serum follicle-stimulating hormone (FSH) levels and menstrual diaries. EP women had an irregular menstrual cycle and FSH $<25 \mathrm{IU} / \mathrm{L}$, while LP women had an irregular cycle and $>25 \mathrm{IU} / \mathrm{L}$. We examined motor evoked potential (MEP) and silent period (SP) elicited by transcranial magnetic stimulation (TMS), in the tibialis anterior muscle at 20\%,40\%, and 60\% of maximal voluntary contraction (MVC) levels, and twitch force potentiation in plantar flexors.

Results: EP group showed a longer SP duration in 40\% MVC condition and larger motor evoked potential amplitude in 20\% MVC condition compared to the LP group. No group difference was detected in twitch force potentiation; however, it correlated negatively with FSH levels. Other factors, such as age, height, body mass index, or physical activity did not explain group differences.

Conclusions: Our preliminary results indicate subtle modulation in both TMS-induced inhibitory and excitatory mechanisms and twitch force potentiation in women already in the late perimenopausal stage. This suggests that the reduction of estrogens may have an accelerating role in the aging process of neuromuscular control.
\end{abstract}

Keywords: Menopause, Follicle-stimulating hormone, Motor cortex, TMS silent period, Twitch force potentiation

\section{Background}

Menopausal transition, i.e. perimenopause, is characterized by a range of physiological changes caused by alterations in female hormone levels [1]. These changes expose women to an early decline in muscle quality and

\footnotetext{
*Correspondence: heidi.m.pesonen@jyu.fi

${ }^{1}$ Faculty of Sport and Health Sciences, University of Jyväskylä, P.O. Box 35, 40014 Jyväskylä, Finland

Full list of author information is available at the end of the article
}

motor function $[2,3]$. Together with changes in muscular properties, neurophysiological and cortical mechanisms play a major role in motor deficits related to the normal aging process $[4,5]$. As changes in muscle function and motor control especially in lower limbs expose individuals to increased risk of falls [6,7], understanding alterations both in muscle function and cortical control is increasingly important. Female sex hormones have a strong neuroprotective role and reduction of estrogens during menopausal transition is associated with multiple 
neurophysiological changes, such as neuroinflammation, mitochondrial dysfunction, and synaptic decline $[8,9]$. While menopause is recognized as a reproductive transition, yet a variety of neural changes are known to occur, and several perimenopausal symptoms are mainly neurological [1].

Transcranial magnetic stimulation (TMS) is a pain-free, non-invasive clinical and therapeutic tool widely used in research to study cortical excitatory and inhibitory mechanisms [10-13]. TMS-elicited silent period (SP) is used to study cortical inhibition and is detected as suppression of on-going activity in surface electromyogram (EMG) of a contracted muscle, following TMS-elicited motor evoked potential (MEP) $[14,15]$. The physiology of SP is under debate, but the early part of the silent period is suggested to originate from spinal mechanisms and the later part from cortical and possibly overlapping spinal inhibitory mechanisms $[12,16]$. SP is believed to represent gamma-aminobutyric acid (GABA) receptormediated inhibitory mechanisms [12]. Pharmacological studies suggest this inhibition arises mostly through type $B$ receptors $\left(\mathrm{GABA}_{\mathrm{B}}\right)[18,19]$. Despite inter-individual variability in SP durations, consistent SP modulation is found in various clinical conditions, such as multiple sclerosis and Parkinson's disease [20, 21].

GABAergic levels are shown to decrease in aging and possibly even more pronounced in aging women [22, 23]. Female sex hormones are known to influence excitatory and inhibitory mechanisms including the GABAergic system [24]. TMS-induced inhibitory mechanisms have been studied in the normal aging process. Aging effects on SP are not entirely clear, however, both a decrease and no change in SP duration have been found in older adults compared to young $[4,25,26]$. Another TMS technique, paired-pulse TMS, has revealed both lengthening and shortening in intracortical inhibition, believed to represent $\mathrm{GABA}_{\mathrm{A}}$ ergic inhibition [27, 28]. Inhibitory and excitatory neuromuscular mechanisms have not been studied during the menopausal transition, despite the changes in muscle function and force production. As menopausal transition seems to accelerate the changes observed during the normal aging process, corticospinal inhibitory mechanisms may demonstrate this modulation early on and thus are of interest in the study of menopause.

Force potentiation is a phenomenon in skeletal muscles, where produced force is temporarily enhanced by recent muscle activity $[29,30]$. Twitch force potentiation is induced by peripheral electrical stimulation before and after conditioning voluntary muscle contraction. The mechanism behind muscle twitch force potentiation is believed to be phosphorylation of myosin regulatory light chains (pRLC), which makes actin and myosin more sensitive to $\mathrm{Ca}^{2+}$ and alters the structure of the myosin head [31]. Another suggested mechanism for force potentiation is the increase in recruitment of higher-order motor units [29]. Twitch force potentiation is shown to be lower in older adults and, interestingly, decreases have been observed already in women aged 45-54 years, about the same age as menopause [32-34]. Estradiol is previously connected to modulation of force potentiation mechanisms and myosin pRLC in mice [35]. Furthermore, Finni et al. [36] found higher twitch torque in postmenopausal women who were users of estrogencontaining hormone replacement therapy (HRT) compared to their monozygotic co-twins, who had never used HRT, without a difference in voluntary force generation. This suggests that modulation of involuntary force generation may be an initial indicator of decline in muscle force in postmenopausal women.

Menopause exposes women to an early decline in physical function and muscle force $[2,3]$. The ability to perform coordinated movements in normal daily living relies on inhibitory and excitatory control. So far, neuromuscular mechanisms have not been investigated in menopausal women. In the present study, we investigated if both TMS-induced SP and peripheral twitch force potentiation are modulated in early and late perimenopausal women, with the changes in hormonal levels already present. The authors hypothesized a modulation in TMS-induced corticospinal inhibitory mechanisms, possibly seen as a shortening of SP, due to changes earlier observed in the aging population [25, 26]. As earlier research shows the modulation in twitch torque in postmenopausal women and modulation of pRLC due to changes in estradiol levels, we also hypothesized a decrease in twitch force potentiation [35, 36]. We recorded neuromuscular data and lower limb functional abilities from 63 women in the early and late stages of perimenopause. We chose lower limbs as our target because of their important role in functional abilities and balance maintenance during the aging process.

\section{Methods \\ Study protocol}

The participants were 63 women aged 48-55 (mean 51.4) years, a representative subgroup of the study population of the Estrogen Regulation of Muscle Apoptosis (ERMA) - project organized at the Gerontology Research Center (GEREC) and the Faculty of Sport and Health Sciences at University of Jyväskylä [37]. The complete ERMA-study protocol, including the current sub-study, was approved by the ethics committee of the Central Finland Health Care District (K-SSHP Dnro 8U/2014), Jyväskylä, Finland. The initial study population was randomly selected from the Finnish National Registry, kept 
by the Population Register Centre, targeting women aged 47 to 55 years living in the Jyväskylä area. An invitation letter with a prequestionnaire and general consent was sent to 6,878 women, from whom $46.9 \%$ responded. A total of 1,627 participants, fitting the inclusion criteria and consented, were invited to the laboratory visit. During the visit, a structured health interview was assessed, fasting blood samples were collected, and participants filled an informed consent for the subsequent phases of the ERMA study. Participants also kept a menstrual diary for at least 12 weeks. Exclusion criteria included estrogen-containing hormonal preparations or other medications affecting ovarian function, current pregnancy or lactation, conditions affecting ovarian function, including bilateral oophorectomy, body mass index $(\mathrm{BMI})>35 \mathrm{~kg} /$ $\mathrm{m}^{2}$ (based on self-reported height and weight), and chronic diseases or medications seriously affecting muscle function. If a participant reported serious or unclear health problems, they were examined by a physician to ensure safe participation in physical performance tests.

The core-ERMA group used in the current study consists of women who had natural reproductive status, i.e., they had an intact uterus and they had not used during the past three months or were not currently using any hormonal contraception or other medication that could affect following up their menstrual bleeding pattern [37]. Menopausal status was determined by measuring participant's follicle-stimulating hormone (FSH) level from a blood sample taken, if possible, during the first five days of the menstrual cycle and by recordings in the menstrual calendar kept for six to twelve months. Also, each participant's $17 \beta$-estradiol (E2) levels were measured. FSH and E2 were detected with immunoassay using IMMULITE 2000 XPi (Siemens Healthcare Diagnostics, UK). All participants who took part in the current study with electrophysiological measurements were perimenopausal, without hormonal contraception, and with an intact uterus. Their perimenopausal status was further defined following Stages of Reproductive Aging Workshop guidelines [38] defined as early perimenopausal (EP) if FSH was below $25 \mathrm{IU} / \mathrm{L}$ and irregular menstrual cycle was reported and late perimenopausal (LP) if FSH was over $25 \mathrm{IU} / \mathrm{L}$. Women with FSH over $30 \mathrm{IU} / \mathrm{L}$ and no menstrual bleeding during the past three to six months were considered postmenopausal and thus they were excluded from the current study. In addition, women with FSH below $17 \mathrm{IU} / \mathrm{L}$ and reporting regular menses were considered premenopausal and thus they also were excluded from the current study. The first three months of menstrual cycle length, the number of bleeding days, and the number of non-bleeding days were calculated for each participant. Three participants from the EP group and four from the LP group did not report any bleeding days during this time.

Women defined to be perimenopausal with a natural hormonal cycle were invited to take part in a subset of functional tests. Ninety-one women volunteered. Researchers performing the measurements and data analysis were blinded to participant's menopausal status and other background information. Good quality electrophysiological data were obtained from 63 participants, from which 25 were EP and 38 were LP (Fig. 1).

\section{Functional measurements}

Physical activity (PA) level was determined by the sevenpoint scale for the current level of weekly leisure-time PA [39]. The scale was previously shown to correlate well with accelerometer-based PA and mobility variables [40]. Questionnaire response categories were: (0) inactive, (1) light activity 1 to 2 times per week, (2) light activity several times per week, (3) moderate activity 1 to 2 times per week, (4) moderate activity several times per week, (5) high activity several times per week, and (6) competitive sports and related training several times per week. Categories 0 and 1 were further combined to low activity group, 2 and 3 to moderate activity group, and 4, 5, and 6 to high activity group.

Maximal isometric knee extension strength was measured in Newton-meters $(\mathrm{Nm})$ in one leg, dominant hand side, with a Good Strength -dynamometer chair (Metitur Oy, Jyväskylä, Finland). The participant's knee was set at $60^{\circ}$ angle from full extension and the ankle was strapped to force transducer. The participant was instructed to extend the knee with maximal force with verbal encouragement. The best performance of three to five isometric extensions was selected for the analysis.

Vertical jumping height was measured three to five times utilizing a contact mat. The jumping height indicates the participant's ability to elevate the body's center of gravity during a vertical countermovement jump. Flight time $(t)$ was measured, and vertical jumping height (m) was calculated: $\left(\mathrm{g} \times \mathrm{t}^{2}\right) / 8 \times 100^{25}$. The highest value was selected for the analysis.

Electrophysiological measurements were performed on the participants' right leg, while she sat in an ankle dynamometer chair, custom made in the University of Jyväskylä [41], with her back and head resting against the backrest. The knee was extended at $180^{\circ}$ and the foot was strapped against the footplate at a $90^{\circ}$ angle. Two surface electrodes $\left(\mathrm{Ambu}^{\circledR}\right.$ BlueSensor N, $22 \times 28 \mathrm{~mm}$, Ballerup, DK) were placed on tibialis anterior (TA) and medial gastrocnemius (MG) muscles for bipolar EMG recording, with a $20 \mathrm{~mm}$ interelectrode distance and a ground electrode placed proximally. 

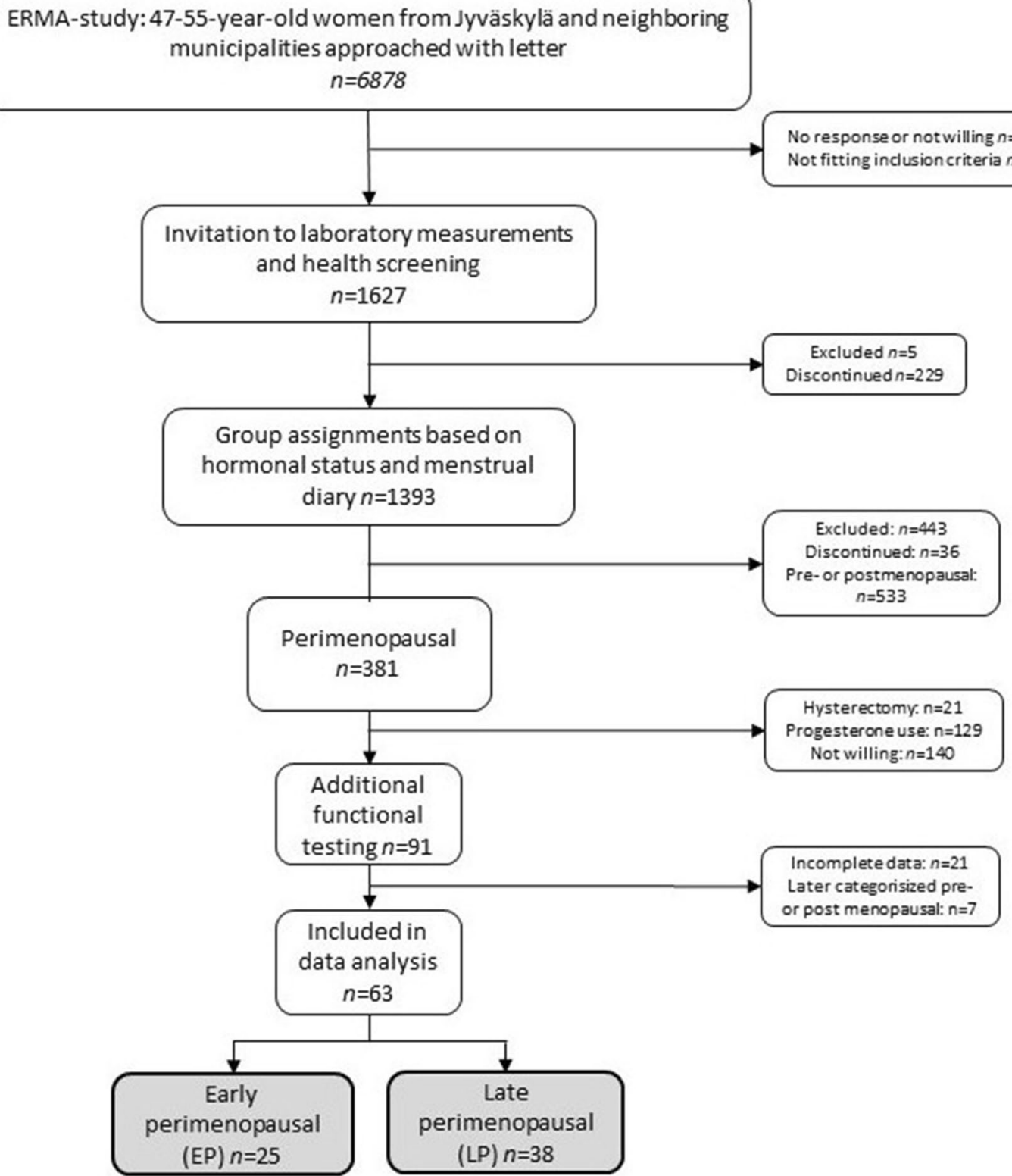

Fig. 1 Flow chart of the recruitment process of the study population

Before TMS procedures, maximal voluntary contraction (MVC) torque for ankle dorsiflexion was determined. MVC was defined as the highest torque from two maximal dorsiflexion contractions and $20 \%, 40 \%$, and $60 \%$ of MVC were calculated. TMS was performed with Magstim Rapid ${ }^{2}$ stimulator (Magstim, Whitland, UK) using a double $70 \mathrm{~mm}$ coil (figure-of-eight coil). Singlepulse stimulation was applied on the motor cortex over the measured and marked center of the scalp, with a $45^{\circ}$ angle from the mid-sagittal lane to target the lower limb representation area on the contralateral hemisphere. Stimulation intensity was increased gradually to find the "hotspot" and assess the resting motor threshold (RMT). Individual RMT in the lower limb was set to the occurrence of $50 \mu \mathrm{V}$ MEP in relaxed TA consecutively three times. Since the lower limb motor cortex locates deep in the central sulcus and may need relatively strong stimulus intensity to elicit MEP, we recorded the used stimulator output for each participant to control for its possible effects. This individual stimulation intensity was used 
while the target muscle was contracted. Participants were instructed to perform isometric dorsiflexion at $20 \%, 40 \%$, and $60 \%$ of their MVC, with rest periods in between. Such submaximal force levels are recommended e.g. by Säisänen et al. [14], as they are relatively easy to maintain during measurement. Produced force and the target force level were shown on the screen in front of the participants. The contraction was held continuously, while 6 to 10 stimulations were delivered at the individual hotspot with 10-s inter-stimulus intervals.

For twitch force recording, peripheral electrical stimulation was delivered to the tibial nerve in the popliteal fossa. The optimal stimulation point was located while the participant was lying prone. Gradually increasing stimulation intensity optimal location and intensity were defined where the peak-to-peak amplitude of the M-wave in the MG muscle and the configuration of the M-wave was repeatable for a minimum of three times. The cathode $\left(\mathrm{Ambu}^{\circledR}\right.$ WhiteSensor $4500 \mathrm{M}, 79 \mathrm{~mm}^{2}$, Ballerup, DK) electrode was placed in this location and the anode electrode (V-trodes; Mettler Electronics, Anaheim, CA, USA) was placed slightly proximal to the patellofemoral joint. Supramaximal electrical stimulation of $1 \mathrm{~ms}$ duration, with $150 \%$ intensity of individual maximal $\mathrm{M}$-wave, was delivered with a constant current stimulator (DS7AH, Digitimer Ltd., Hertfordshire, UK). The participant was then seated in the dynamometer chair and instructed to relax, while the first stimulus was delivered, and after that to perform a maximal isometric plantarflexion, during which the second stimulus was delivered. After 2-5 s relaxation, the third stimulus was delivered to a relaxed muscle. Participants received verbal encouragement to perform the contraction and visual feedback of their torque level from the screen. Three trials including three stimulations were performed with 60 -s rest intervals in between.
EMG signals were amplified $1000 \times$ and band-pass filtered $(10 \mathrm{~Hz}-1 \mathrm{kHz})$. The ankle MVC was measured with a torque-transducer (Kistler Group, Switzerland), mounted between the ergometer servomotor and the platform of the foot.

\section{Data analysis}

For TMS data, individual MEP and SP responses were analyzed manually (Spike2 version 6.17, Cambridge Electronics Design, Cambridge, UK). MEP-start was detected as the point where the TA EMG signal exceeded baseline activity and the MEP-end as the point where the complete EMG silence began. This same time-point was also the SP-start. MEP amplitude was determined as the largest peak-to-peak amplitude. SP-end was where baseline EMG activity returned. For statistical analysis, both "absolute" SP duration (SP-start - SP-end) and "relative" SP (MEP-start - SP-end) were recorded [14, 42]. If a participant had four or more successful MEPs and SPs in one force level, those with the shortest and longest durations were not included in further analysis. From the remaining recordings, mean values for SP and MEP for each participant were calculated for each force level.

The electrical stimulation data were analyzed using Matlab (R2015a, 8.5.0, Mathworks Inc., Natick, MA, USA). Peak twitch torque (PTT) and maximal voluntary contraction (MVC) torque were manually detected from the torque signal (Fig. 2). Twitch force potentiation was analyzed by comparing PTT of pre-MVC twitch peak amplitude to post-MVC twitch peak amplitude in the recorded three trials using the equation: twitch potentiation $(\%)=(($ post-MVC PTT/pre-MVC PTT $) /$ pre-MVC PTT) $\times 100$. The best potentiation effect and the best result from MVC out of three trials were selected for further analysis.

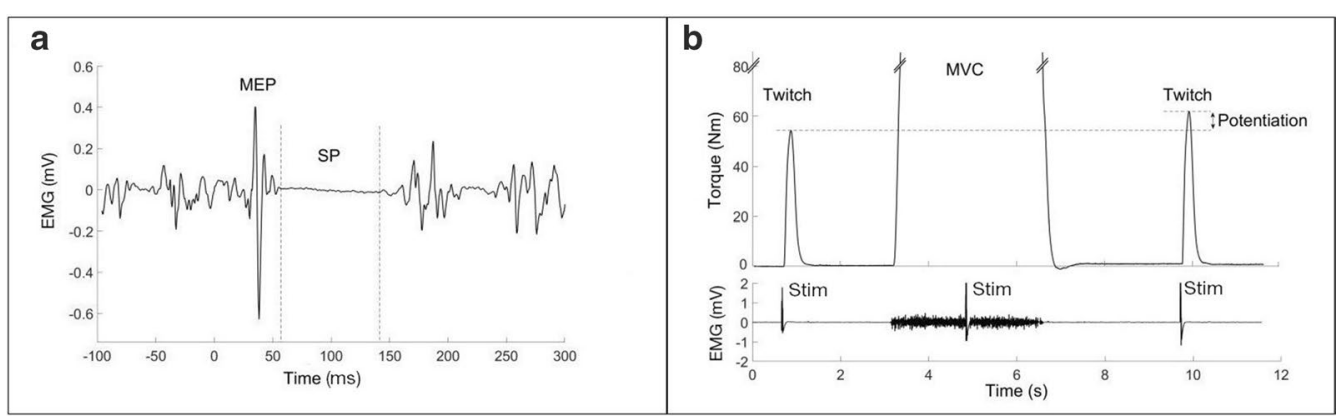

Fig. 2 A. EMG recording of TMS response from a single participant at 40\% MVC condition in tibialis anterior muscle. Dashed lines indicate the start and end of SP. MEP amplitude was calculated as the maximum peak-to-peak amplitude. B. Torque data of twitch force potentiation analysis is presented from a single participant. Potentiation was calculated as the difference between pre-MVC twitch and post-MVC twitch. Stimulus artifacts are detectable in the EMG recording from the medial gastrocnemius muscle 
Statistical analysis was performed with IBM SPSS Statistics Version 24 (IBM Corporation, Chicago, IL, USA). Group comparisons between EP and LP women were performed with independent samples t-tests and for PA variable with chi-square test. Variability between different stimulation conditions was tested with pairedsample t-test. Correlations were detected with Pearson correlation coefficient or ANOVA. Covariance was tested with ANCOVA. The significance level was set to $\mathrm{p}<0.05$.

Table 1 Characteristics of the participants, mean ( \pm SD) or $n(\%)$

\begin{tabular}{llll}
\hline & $E P(n=25)$ & $L P(n=38)$ & P-value \\
\hline Age, y & $51.0( \pm 2.0)$ & $51.6( \pm 1.8)$ & 0.222 \\
Height, cm & $163.8( \pm 0.1)$ & $164.2( \pm 0.1)$ & 0.810 \\
BMI & $25.4( \pm 4.4)$ & $25.3( \pm 3.7)$ & 0.916 \\
FSH (IU/L) & $17.32( \pm 4.79)$ & $48.25( \pm 22.28)$ & $<0.001$ \\
E2 (nmol/L) & $0.374( \pm 0.257)$ & $0.249( \pm 0.200)$ & 0.035 \\
Mean cycle length & $36.8( \pm 22.2)^{a}$ & $65.8( \pm 41.7)^{\mathrm{b}}$ & 0.001 \\
Mean bleeding days & $6.0( \pm 1.4)^{\mathrm{a}}$ & $5.3( \pm 1.5)^{\mathrm{b}}$ & 0.098 \\
Mean non-bleeding days & $34.7( \pm 31.6)^{\mathrm{a}}$ & $65.2( \pm 49.1)^{\mathrm{b}}$ & 0.006 \\
Physical activity & & & 0.652 \\
Low & $4(16)$ & $6(16)$ & \\
Moderate & $7(28)$ & $7(18)$ & 0.691 \\
High & $14(56)$ & $25(66)$ & $84.5( \pm 9,6)$ \\
Stimulus intensity (\%) & $83.6( \pm 7.7)$ & &
\end{tabular}

P-values tested with independent samples t-test, physical activity level with chi square test. $\mathrm{BMI}=$ body mass index, $\mathrm{cm}=$ centimeter, $\mathrm{E} 2=17 \beta$-estradiol, $\mathrm{FSH}=$ follicle-stimulating hormone, IU/L = international units per liter, $\mathrm{nmol} / \mathrm{L}=$ nanomoles per liter, $\mathrm{SD}=$ standard deviation, $\mathrm{y}=$ year. ${ }^{\mathrm{a}} \mathrm{n}=23,{ }^{\mathrm{b}} \mathrm{n}=34$.

\section{Results}

\section{Group characteristics}

Both groups were approximately the same age, height, and BMI (see Table 1). As expected, the EP group had lower FSH levels and higher E2 levels than the LP group as well as a shorter duration of the menstrual cycle for the first three months of the follow-up period. No differences were detected in PA habits between groups. Stimulus intensities varied across participants but there were no differences between groups.

\section{Silent period}

SP duration differed between EP and LP groups in $40 \%$ MVC condition $(\mathrm{t}(61)=2.473, \mathrm{p}=0.016)$. Mean absolute SP duration for the EP group was $57.1 \pm 25.8 \mathrm{~ms}$ and for the LP group $42.8 \pm 20.1 \mathrm{~ms}$. The difference remained after controlling for stimulus intensity $(\mathrm{F}(1,60)=6.044, \mathrm{p}=0.017)$. There was no significant difference between groups in $20 \% \mathrm{MVC}$ $(47.8 \pm 22.0 / 41.1 \pm 22.7, \mathrm{t}(61)=1.156, \mathrm{p}=0.252)$ and $60 \%$ MVC $(62.2 \pm 33.6 / 51.3 \pm 24.5, \mathrm{t}(61)=1.491, \mathrm{p}=0.141)$ conditions (Fig. 3). Duration of SP increased with required muscle force for all participants $(\mathrm{p}<0.01)$. In EP group the increase in SP duration was significant from 20 to $40 \%$ MVC conditions $(\mathrm{p}<0.01)$ and in LP group from 40 to $60 \%$ MVC conditions ( $\mathrm{p}<0.01$ ) (Fig. 3).

When analyzing relative SP, it also differed between groups in $40 \%$ MVC condition $(\mathrm{t}(61)=2.494$, $\mathrm{p}=0.015)$. Mean relative SP duration for EP group was $88.4 \pm 29.4 \mathrm{~ms}$ and for LP group $72.1 \pm 22.5 \mathrm{~ms}$. No significant difference was found in $20 \%$ MVC $(78.4 \pm 25.6 \mathrm{~ms} / 68.7 \pm 25.5 \quad \mathrm{~ms}, \quad \mathrm{t}(61)=1.475$, $\mathrm{p}=0.145)$ or $60 \% \mathrm{MVC}(93.2 \pm 38.1 \mathrm{~ms} / 80.9 \pm 26.9 \mathrm{~ms}$, $\mathrm{t}(61)=1.507, \mathrm{p}=0.137)$ conditions.
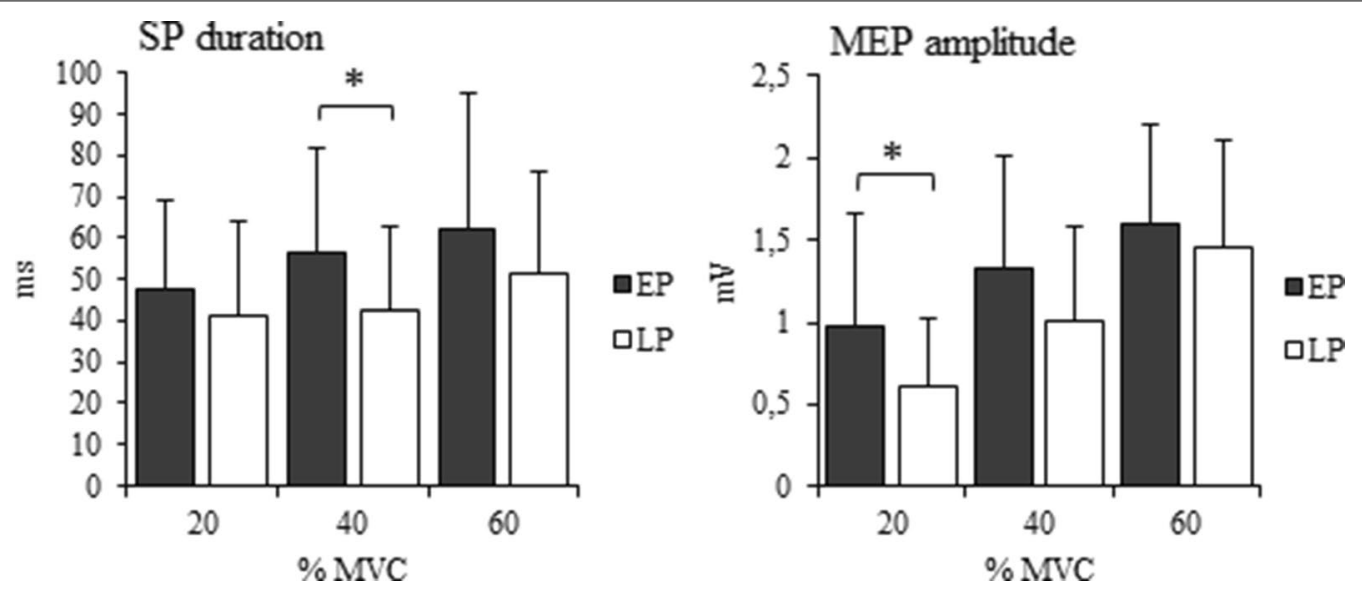

Fig. 3 Mean SP duration and MEP amplitude ( $m$ s and $m V$, error bar indicates SD) in EP $(n=25)$ and LP ( $n=38)$ groups, recorded in all three (20\%, $40 \%$ and $60 \%$ MVC) conditions. * indicates significant difference $(p<.05)$ 


\section{Motor evoked potential}

MEP amplitude differed between groups in 20\% MVC condition $(\mathrm{t}(61)=2.511, \mathrm{p}=0.017)$. EP group mean amplitude was $1.000 \pm 0.688 \mathrm{mV}$ and for LP group $0.617 \pm 0.409 \mathrm{mV}$. The difference remained when controlling for stimulus intensity $(\mathrm{F}(1,60)=7.460, \mathrm{p}=0.008)$. MEP amplitudes showed a similar tendency in $40 \%$ MVC condition, as mean amplitude was $1.326 \pm 0.707 \mathrm{mV}$ in EP group and $1.010 \pm 0.576 \mathrm{mV}$ in LP group $(\mathrm{t}(61)=1.944$, $\mathrm{p}=0.057$ ), albeit not significant. There was no significant difference in MEP amplitudes in $60 \%$ MVC condition $(1.598 \pm 0.607 \mathrm{mV} / 1.454 \pm 0.648 \mathrm{mV}, \mathrm{t}(62)=0.883$, $\mathrm{p}=0.381)$. MEP amplitude increased with muscle force in both groups $(\mathrm{p}<0.001)$.

Absolute SP duration and MEP amplitude correlated in $40 \%$ MVC condition $(\mathrm{r}=0.260, \mathrm{p}=0.040)$ (Fig. 4) and $20 \%$ MVC condition showed similar tendency, but not significant $(\mathrm{r}=0.235, \mathrm{p}=0.063)$. No correlation was found between SP duration and MEP amplitude in $60 \%$ MVC condition $(r=0.077, p=0.548)$.

\section{Twitch force potentiation and physical performance}

Twitch force potentiation did not differ between groups. An association between twitch force potentiation and FSH $(r=-0.262, p=0.043)$ was observed. No association was found between twitch force potentiation and E2 $(r=0.123, p=0.348)$. There were no differences between groups in vertical jumping height, knee extension strength, or ankle dorsiflexion strength (Table 2). However, EP group had $17.2 \%$ stronger plantar flexion compared to LP group $(t(57)=2.533, p=0.014)$. There was no correlation between plantar flexion torque and SP duration in $40 \% \mathrm{MVC}$ condition $(\mathrm{r}=0.029, \mathrm{p}=0.830)$ nor

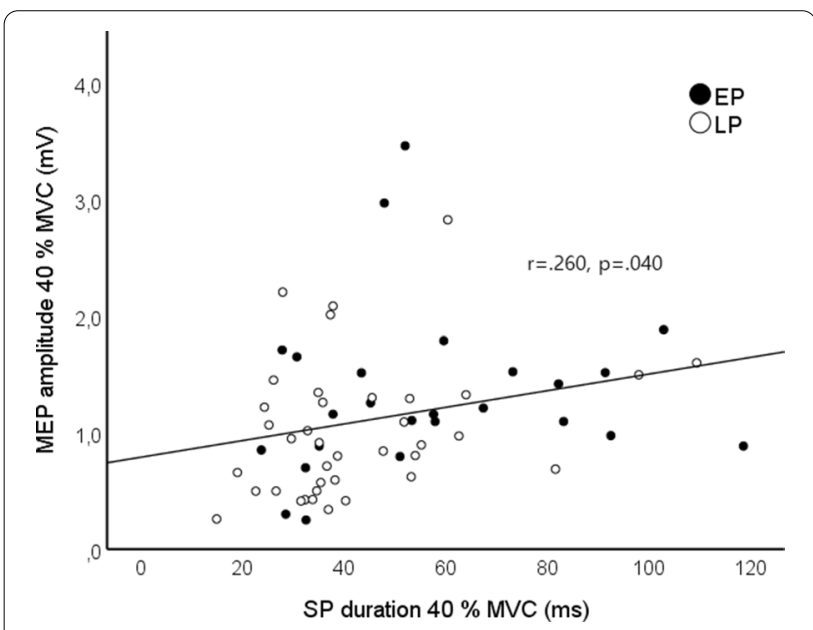

Fig. 4 Association between mean MEP amplitude $(\mathrm{mV})$ and SP duration (ms) in 40\% MVC condition. Black circles represent the EP group $(n=25)$ and white circles the LP group $(n=38)$
Table 2 Measures of lower limb physical performance and twitch force potentiation, mean $( \pm S D$ )

\begin{tabular}{llll}
\hline & $E P(n=25)$ & $L P(n=38)$ & P-value \\
\hline MVC dorsiflexion (Nm) & $31.9( \pm 9.1)$ & $34.3( \pm 6.7)$ & 0.238 \\
MVC plantarflexion (Nm) & $131.1( \pm 33.9)^{\mathrm{b}}$ & $108.8( \pm 32.4)^{\mathrm{b}}$ & 0.014 \\
$\begin{array}{c}\text { Knee extension strength } \\
(\mathrm{Nm})\end{array}$ & $162.2( \pm 27.9)^{\mathrm{b}}$ & $153.7( \pm 38.8)^{\mathrm{c}}$ & 0.360 \\
$\begin{array}{l}\text { Vertical jumping height }(\mathrm{m}) \\
\begin{array}{c}\text { Twitch force potentiation } \\
(\%)\end{array}\end{array}$ & $0.185( \pm 0.046)^{\mathrm{a}}$ & $0.200( \pm 0.037)^{\mathrm{a}}$ & 0.177 \\
& $10.1( \pm 6.3)^{\mathrm{a}}$ & $6.7( \pm 9.3)^{\mathrm{b}}$ & 0.124 \\
\hline
\end{tabular}

P-values tested with independent samples t-test. MVC $=$ maximal voluntary contraction, $\mathrm{m}=$ meter, $\mathrm{Nm}=$ Newton meter, $\mathrm{SD}=$ standard deviation.

${ }^{a} n=$ missing one, ${ }^{b} n=$ missing two, ${ }^{c} n=$ missing five.

with plantarflexion torque and MEP amplitude in 20\% condition $(\mathrm{r}=0.130, \mathrm{p}=0.327)$.

\section{Discussion}

The aim of the present study was to examine TMSinduced inhibition and peripheral twitch force potentiation in women in early and late perimenopausal stages, to determine if the progression of the menopausal stage affects motor control mechanisms. Our finding was that women in the EP group presented longer SP durations than women in the LP during a moderate force production condition (40\% MVC). MEP amplitudes were sensitive to the perimenopausal stage as in 20\% MVC condition EP group showed larger MEP amplitudes and the same tendency remained in $40 \%$ MVC condition. Furthermore, an association was observed between twitch force potentiation and FSH. Our participants were comparable in age, height, BMI, and PA level and thus those factors do not explain the differences we detected.

Our results indicate a reduction in corticospinal inhibitory mechanisms observable already in late perimenopause. While pharmacological studies have provided strong evidence for GABAergic origins of SP [17-19, 43], there remains a debate over the spinal and cortical contribution to the generation of SP. Formerly, the early part ( $~ 50 \mathrm{~ms}$ ) of SP has been thought to arise from spinal origins and the later part from cortical origins [16-19, 42, 44]. Recently Yacyshyn et al. [45] suggested that spinal inhibitory mechanisms could play an even larger role in SP generation than previously thought. They found cervicomedullary motor evoked potential suppression at up to $150 \mathrm{~ms}$ after TMS. However, as long-lasting cortical GABAergic neurons probably affect SP throughout its duration, it is unclear how the spinal and cortical inhibitory mechanisms interact in the latter part of the SP. Further studies are needed to determine their roles in SP generation. 
During aging, competencies indicating good motor coordination and performance are important, as they reflect an individual's overall motor abilities and the risk of falls [6, 7]. This association is further emphasized in aging women, as menopause exposes them to early decline of muscle function [2, 3]. TMS studies indicate a strong cortical contribution in controlling muscle activity e.g. during the gait cycle, that can be modulated by intracortical inhibition [46]. There is also growing evidence that older individuals utilize somewhat different cortical mechanisms in motor control tasks compared to young $[47,48]$. Along with decreasing levels of GABA [22, 23], decreased inhibition has been observed in older adults, and it has been connected to decreased control of coordinated movements $[25,26,49,50]$. Recently, Swanson and Fling [50, 51] reported shorter SP in older adults than young. Older adults also demonstrated an association between shorter SP and reduced performance in walking and turning, while an opposite correlation was observed for young adults. This was only present in the right hemisphere stimulation. The results suggest that decreased inhibitory control may underlie age-related decline in motor control and coordination. Our results offer a proposal that changes in hormonal levels in menopausal transition may accelerate the decrease in inhibitory mechanisms in cortical or spinal levels and possibly accelerate the aging process of motor function. Unlike the study by Swanson \& Fling [50], we performed the stimulation only to the left hemisphere, where they did not find significant differences between young and older adults. Therefore, we cannot make further conclusions about hemispheric differences.

Furthermore, we found lower MEP amplitude in LP than in the EP group indicating modulation of cortical excitability in perimenopausal stages. Reduced MEP amplitudes in older adults compared to young are a common finding, and our results suggest that menopause may accelerate this modulation [25, 26, 49]. Here, SP durations and MEP amplitudes were associated in moderate force production condition. This relationship between SP and MEP has been previously reported [14, 25, 52]. Therefore, the mechanisms that underlie the changes observed in SP and MEP, may have similar origins.

Twitch force potentiation did not differ between EP and LP groups. However, there was a negative correlation between participants' FSH level and twitch force potentiation. This correlation suggests modulation toward decreasing twitch force potentiation in the menopausal transition. FSH level fluctuates also during menstrual cycle in women. However, no association has been found earlier with involuntary muscle contractile properties and FSH levels during menstrual cycle [53]. A correlation was not present with E2 but only with FHS, which may be more sensitive here to different stages of perimenopause. Furthermore, the very subtle fluctuations of E2 may not be detected with IMMULITE $2000 \mathrm{XPi}$. Twitch force potentiation and twitch torque have been shown to decrease in aging women and are suggested to partly underlie the morphological and functional changes observed in aged muscle $[33,36]$. As estradiol seems to modulate force potentiation mechanisms [35], it may be that the twitch force potentiation deteriorates in menopausal women, but this modulation was not large enough to be yet detected between the perimenopausal stages in the present study.

There were no differences between groups in knee extension strength, vertical jumping height, or ankle dorsiflexion strength. Our results suggest that these functional measures are not yet sensitive for a decline in present perimenopausal stages. Nevertheless, plantar flexion strength was higher in EP group than LP group and may point toward early modulation in muscle function in menopause. it should be noted that the decrease in hormonal levels in menopause is a risk factor for sarcopenia. It seems that the late perimenopausal stage does not yet reveal evidence for a decrease in muscle strength and function, although direction toward it may be observed [54].

This study has several limitations. Our sample size is relatively small, and the observed interactions should therefore be investigated in larger samples. TMS stimulation was performed without advanced navigation and information on individual brain images. Leg motor areas are relatively small, located deep in the inter-hemispheric fissure and the optimal stimulation points are less segregated than those of hand muscles [55]. Therefore, it can be challenging to find the optimal stimulation point and higher intensities may be required to elicit measurable MEP compared to hand [56]. For this, we used a stimulus intensity that was the individually detected lower limb RMT for each participant. This may provide a challenge for generalization, as different levels of stimulation intensities have resulted in different results in research. E.g. changes in SP after fatiguing exercise have been detected at lower TMS intensities but not at higher intensities [57]. It might be that the changes detected here are present at low stimulus intensities but not with higher ones. However, this requires further investigation. As the detection of RMT and SP may be challenging for the lower limb and older participants, we controlled our results for the intensity of the stimulator output. Furthermore, the number of TMS trials to achieve each MEP and SP average was rather small $[12,58]$. Unlike the study by Swanson \& Fling [50], we performed the stimulation only to the left hemisphere, where they did not find significant differences between young and older adults. Therefore, 
we cannot make further conclusions about hemispheric differences. Thus, our preliminary results require further investigation.

Our method to measure very low E2 levels with IMMULITE $2000 \mathrm{XPi}$ has some inaccuracy, and thus the most subtle fluctuations may not be revealed. Therefore, all associations may not have become apparent. Our results of E2 levels were in line with FSH levels and menstrual histories. Our study participants did not include pre- or postmenopausal women; thus, we cannot draw inferences of how the detected modulation in inhibitory or excitatory processes relates to either the fertility stage or the postmenopausal stage.

\section{Conclusions}

Our preliminary results indicate subtle modulation toward decreasing TMS-induced inhibition in the central nervous system and possibly decreasing muscle twitch force potentiation in perimenopausal women. Faultless interaction of inhibitory and excitatory processes is essential in appropriate motor control and our results suggest that the reduction of estrogens in menopausal transition may accelerate the aging process related to this interaction.

\begin{abstract}
Abbreviations
ANOVA: One-way analysis of variance; ANCOVA: Analysis of covariance; BMI: Body mass index; E2: 17ß-Estradiol; EMG: Electromyography; EP: Early perimenopausal; FSH: Follicle-stimulating hormone; GABA: Gamma-aminobutyric acid; LP: Late perimenopausal; MEP: Motor evoked potential; MG: Medial gastrocnemius; MVC: Maximal voluntary contraction; Nm: Newton meters; pRLC: Phosphorylation of myosin regulatory light chains; PA: Physical activity; PTT: Peak twitch torque; RMT: Resting motor threshold; SD: Standard deviation; SP: Silent period; TA: Tibialis anterior; TMS: Transcranial magnetic stimulation.
\end{abstract}

\section{Acknowledgements}

The authors would like to thank the study participants and the personnel of the Faculty of Sport and Health Sciences, University of Jyväskylä.

\section{Authors' contributions}

EL, VK, SS, TF and IMT conceived and designed research. HP, EL, PH and SS performed experiments. HP, EL, PA, SS and TF analyzed data. HP, EL, PA, TF and IMT interpreted results of experiments. HP prepared figures and drafted manuscript. HP, EL, PH, PA, VK, SS, TF and IMT edited and revised manuscript. All authors approved final version of manuscript.

\section{Funding}

This study received funding from Academy of Finland (No. 275323, Vuokko Kovanen \& no. 309504, Eija K. Laakkonen) and Juho Vainion säätiö (2016-2018, Eija K. Laakkonen).

\section{Availability of data and materials}

The datasets used and analyzed during the current study are available from the corresponding author on reasonable request.

\section{Declarations}

Ethics approval and consent to participate

The complete ERMA-study protocol, including the current sub study, was approved by the ethics committee of the Central Finland Health Care District
(K-SSHP Dnro 8U/2014), Jyväskylä, Finland. All participants filled an informed consent for the complete ERMA study and subsequent phases.

\section{Consent for publication}

Not applicable.

\section{Competing interests}

The authors declare that they have no competing interests.

\section{Author details}

1 Faculty of Sport and Health Sciences, University of Jyväskylä, P.O. Box 35, 40014 Jyväskylä, Finland. ${ }^{2}$ Gerontology Research Center, University of Jyväskylä, Jyväskylä, Finland. ${ }^{3}$ Department of Obstetrics and Gynecology, Pelvic Floor Research and Therapy Unit, Central Finland Central Hospital, Jyväskylä, Finland.

Received: 11 September 2020 Accepted: 21 March 2021

Published online: 31 March 2021

\section{References}

1. Brinton RD, Yao J, Yin F, et al. Perimenopause as a neurological transition state. Nat Rev Endocrinol. 2015;11(7):393-405.

2. Sipilä S, Narici M, Kjaer M, et al. Sex hormones and skeletal muscle weakness. Biogerontology. 2013;14(3):231-45.

3. Bondarev D, Laakkonen EK, Finni T, et al. Physical performance in relation to menopause status and physical activity. Menopause. 2018;25(12):1432-41.

4. Bhandari A, Radhu N, Farzan F, et al. A meta-analysis of the effects of aging on motor cortex neurophysiology assessed by transcranial magnetic stimulation. Clin Neurophysiol. 2016;127(8):2834-45.

5. Ward NS, Swayne OBC, Newton JM. Age-dependent changes in the neural correlates of force modulation: An fMRI study. Neurobiol Aging. 2008:29(9):1434-46.

6. de Rekeneire N, Visser M, Peila R, et al. Is a fall just a fall: Correlates of falling in healthy older persons. The health, aging and body composition study. J Am Geriatr Soc. 2003;51(6):841-6.

7. Kannus P, Palvanen $M$, Niemi $S$, et al. Alarming rise in the number and incidence of fall-induced cervical spine injuries among older adults. J Gerontol A Biol Sci Med Sci. 2007;62(2):180-3.

8. Marin R, Diaz M. Estrogen interactions with lipid rafts related to neuroprotection. Impact of brain ageing and menopause. Front Neurosci. 2018;12:128.

9. Zarate S, Stevnsner T, Gredilla R. Role of estrogen and other sex hormones in brain aging. Neuroprotection and DNA repair. Front Aging Neurosci. 2017;9:430

10. Lefaucheur J, André-Obadia N, Antal A, et al. Evidence-based guidelines on the therapeutic use of repetitive transcranial magnetic stimulation (rTMS). Clin Neurophysiol. 2014;125(11):2150-206.

11. Lefaucheur J, Picht T. The value of preoperative functional cortical mapping using navigated TMS. Clin Neurophysiol. 2016;46(2):125-33.

12. Rossini PM, Burke D, Chen R, et al. Non-invasive electrical and magnetic stimulation of the brain, spinal cord, roots and peripheral nerves: basic principles and procedures for routine clinical and research application. An updated report from an IFCN Committee. Clin Neurophysiol. 2015;126(6):1071-107.

13. Tarkka IM, Könönen M, Pitkänen K, et al. Alterations in cortical excitability in chronic stroke after constraint-induced movement therapy. Neurol Res. 2008;30(5):504-10.

14. Säisänen L, Pirinen E, Teitti S, et al. Factors influencing cortical silent period: optimized stimulus location, intensity and muscle contraction. J Neurosci Methods. 2008;169(1):231-8.

15. Inghilleri M, Berardelli A, Cruccu G, et al. Silent period evoked by transcranial stimulation of the human cortex and cervicomedullary junction. J Physiol. 1993;466:521-34.

16. Chen R, Lozano AM, Ashby P. Mechanism of the silent period following transcranial magnetic stimulation. Evidence from epidural recordings. Exp Brain Res. 1999;128(4):539-42.

17. Pierantozzi M, Marciani MG, Palmieri MG, et al. Effect of Vigabatrin on motor responses to transcranial magnetic stimulation: an effective tool 
to investigate in vivo GABAergic cortical inhibition in humans. Brain Res. 2004;1028(1):1-8.

18. Stetkarova I, Kofler M. Differential effect of baclofen on cortical and spinal inhibitory circuits. Clin Neurophysiol. 2013;124(2):339-45.

19. Werhahn KJ, Kunesch E, Noachtar S, et al. Differential effects on motorcortical inhibition induced by blockade of GABA uptake in humans. J Physiol. 1999:517(Pt 2):591-7.

20. Caramia MD, Palmieri MG, Desiato MT, et al. Brain excitability changes in the relapsing and remitting phases of multiple sclerosis: a study with transcranial magnetic stimulation. Clin Neurophysiol. 2004;115(4):956-65.

21. Kojovic M, Kassavetis P, Bologna M, et al. Transcranial magnetic stimulation follow-up study in early Parkinson's disease: A decline in compensation with disease progression? Mov Disord. 2015;30(8):1098-106.

22. Gao F, Edden RA, Li M, et al. Edited magnetic resonance spectroscopy detects an age-related decline in brain GABA levels. Neuroimage. 2013;78:75-82.

23. Hermans $L$, Leunissen I, Pauwels $L$, et al. Brain GABA levels are associated with inhibitory control deficits in older adults. J Neurosci. 2018;38(36):7844-51.

24. Barth C, Villringer A, Sacher J. Sex hormones affect neurotransmitters and shape the adult female brain during hormonal transition periods. Front Neurosci. 2015:9:37.

25. Sale MV, Semmler JG. Age-related differences in corticospinal control during functional isometric contractions in left and right hands. J Appl Physiol. 2015;99(4):1483-93.

26. Oliviero A, Profice P, Tonali PA, et al. Effects of aging on motor cortex excitability. Neurosci Res. 2006;55(1):74-7.

27. Peinemann A, Lehner C, Conrad B, Siebner HR. Age-related decrease in paired-pulse intracortical inhibition in the human primary motor cortex. Neurosci Lett. 2001;313(1-2):33-6.

28. McGinley M, Hoffman RL, Russ DW, et al. Older adults exhibit more intracortical inhibition and less intracortical facilitation than young adults. Exp Gerontol. 2010:45(9):671-8.

29. Tillin NA, Bishop D. Factors modulating post-activation potentiation and its effect on performance of subsequent explosive activities. Sports Med. 2009;39(2):147-66.

30. Miller JD, Herda TJ, Trevino MA, et al. Time-related changes in firing rates are influenced by recruitment threshold and twitch force potentiation in the first dorsal interosseous. Exp Physiol. 2017;102(8):950-61.

31. Szczesna $D$, Zhao J, Jones $M$, et al. Phosphorylation of the regulatory light chains of myosin affects $\mathrm{Ca}^{2+}$ sensitivity of skeletal muscle contraction. J Appl Physiol. 2002;92(4):1661-70.

32. Kuu S, Gapeyeva H, Ereline J, et al. Twitch contractile properties of plantarflexor muscles in young and middle-aged recreationally physically active and non-active women. Aging Clin Exp Res. 2007;19(1):48-54.

33. Miller JD, Herda TJ, Trevino MA, et al. Age-related differences in twitch properties and muscle activation of the first dorsal interosseous. Clin Neurophysiol. 2017;128(6):925-34

34. Schoenaker DA, Jackson CA, Rowlands JV, et al. Socioeconomic position, lifestyle factors and age at natural menopause: a systematic review and meta-analyses of studies across six continents. Int J Epidemiol. 2014;43(5):1542-62

35. Lai S, Collins BC, Colson BA, et al. Estradiol modulates myosin regulatory light chain phosphorylation and contractility in skeletal muscle of female mice. Am J Physiol Endocrinol Metab. 2016:310(9):724.

36. Finni T, Noorkoiv M, Pöllänen $E$, et al. Muscle function in monozygotic female twin pairs discordant for hormone replacement therapy. Muscle Nerve. 2011:44(5):769-75.

37. Kovanen V, Aukee P, Kokko K, et al. Design and protocol of Estrogenic Regulation of Muscle Apoptosis (ERMA) study with 47 to 55 -year-old women's cohort: novel results show menopause-related differences in blood count. Menopause. 2018:25(9):1020-32.

38. Harlow SD, Gass M, Hall JE, et al. Executive summary of the Stages of Reproductive Aging Workshop + 10: addressing the unfinished agenda of staging reproductive aging. J Clin Endocrinol Metab. 2012:97(4):1159-68

39. Hirvensalo M, Lampinen P, Rantanen T. Physical exercise in old age: An eight-year follow-up study on involvement, motives and obstacles among persons age 65-84. J Aging Phys Act. 1998;6:157-68.
40. Hyvarinen M, Sipila S, Kulmala J, et al. Validity and reliability of a single question for leisure-time physical activity assessment in middle-aged women. J Aging Phys Act. 2019;1-11.

41. Peltonen J, Cronin NJ, Avela J, et al. In vivo mechanical response of human Achilles tendon to a single bout of hopping exercise. J Exp Biol. 2010;213(Pt 8):1259-65

42. Stetkarova I, Leis AA, Stokic DS, et al. Characteristics of the silent period after transcranial magnetic stimulation. Am J Phys Med Rehabil. 1994:73(2):98-102.

43. Siebner HR, Dressnandt J, Auer C, et al. Continuous intrathecal baclofen infusions induced a marked increase of the transcranially evoked silent period in a patient with generalized dystonia. Muscle Nerve. 1998:21(9):1209-12

44. Ziemann U, Netz J, Szelenyi A, et al. Spinal and supraspinal mechanisms contribute to the silent period in the contracting soleus muscle after transcranial magnetic stimulation of human motor cortex. Neurosci Lett. 1993;156(1-2):167-71.

45. Yacyshyn AF, Woo EJ, Price MC, et al. Motoneuron responsiveness to corticospinal tract stimulation during the silent period induced by transcranial magnetic stimulation. Exp Brain Res. 2016;234(12):3457-63.

46. Petersen NT, Butler JE, Marchand-Pauvert V, et al. Suppression of EMG activity by transcranial magnetic stimulation in human subjects during walking. J Physiol. 2001;537(Pt 2):651-6.

47. Heuninckx S, Wenderoth N, Swinnen SP. Systems neuroplasticity in the aging brain: recruiting additional neural resources for successful motor performance in elderly persons. J Neurosci. 2008;28(1):91-9.

48. Mattay VS, Fera F, Tessitore A, et al. Neurophysiological correlates of agerelated changes in human motor function. Neurology. 2002;58(4):630-5

49. Fujiyama $\mathrm{H}$, Hinder MR, Schmidt MW, et al. Age-related differences in corticospinal excitability and inhibition during coordination of upper and lower limbs. Neurobiol Aging. 2012;33(7):1484.e1-14.

50. Swanson CW, Fling BW. Associations between gait coordination, variability and motor cortex inhibition in young and older adults. Exp Gerontol. 2018;113:163-72.

51. Swanson CW, Fling BW. Associations between Turning Characteristics and Corticospinal Inhibition in Young and Older Adults. Neuroscience. 2020;425:59-67.

52. Taylor JL, Allen GM, Butler JE, et al. Effect of contraction strength on responses in biceps brachii and adductor pollicis to transcranial magnetic stimulation. Exp Brain Res. 1997:117(3):472-8.

53. JansedeJonge $X A, B o o t C R$, Thom JM, et al. The influence of menstrual cycle phase on skeletal muscle contractile characteristics in humans. J Physiol. 2001;530(Pt 1):161-6.

54. Sipila S, Tormakangas T, Sillanpaa E, et al. Muscle and bone mass in middle-aged women: role of menopausal status and physical activity. J Cachexia Sarcopenia Muscle. 2020;11(3):698-709.

55. Niskanen E, Julkunen P, Säisänen L, et al. Group-level variations in motor representation areas of thenar and anterior tibial muscles: Navigated Transcranial Magnetic Stimulation Study. Hum Brain Mapp. 2010;31(8):1272-80

56. Kesar TM, Stinear JW, Wolf SL. The use of transcranial magnetic stimulation to evaluate cortical excitability of lower limb musculature: challenges and opportunities. Restor Neurol Neurosci. 2018:36(3):333-48.

57. Temesi J, Rupp T, Martin V, et al. Central fatigue assessed by transcranial magnetic stimulation in ultratrail running. Med Sci Sports Exerc. 2014;46(6):1166-75

58. Škarabot J, Mesquita RNO, Brownstein CG, Ansdell P. Myths and Methodologies: How loud is the story told by the transcranial magnetic stimulation-evoked silent period? Exp Physiol. 2019;104(5):635-42.

\section{Publisher's Note}

Springer Nature remains neutral with regard to jurisdictional claims in published maps and institutional affiliations. 